\title{
sciendo
}

\author{
AGNIESZKA M. GRZEBALSKA, ANNA STEĆ, IZABELA ŁAWNICKA, \\ ANNA BEDNAREK-SKUBLEWSKA, ANDRZEJ KSIAZŻEK
}

\section{Retrospective single-center analysis of the reasons and serious complications of peritoneal-related peritonitis in patients with end-stage renal disease undergoing peritoneal dialysis treatment}

\begin{abstract}
Introduction. Peritonitis is still a serious complication of peritoneal dialysis (PD). Consequences of peritonitis can be severe. The most severe are peritoneal dialysis discontinuation and patient's death. In majority, peritonitis is bacterial in the origin. Mainly there is a gram-positive infection, less commonly gram-negative one. Some peritonitis are culture-negative, because of former antibiotics use. In minority, fungal, tuberculous or even viral peritonitis are observed.

Aim. The aim of the present study is to analyze the number, origin and serious complications of peritoneal-related peritonitis cases found in our PD center.

Material and methods. We performed a retrospective five-years evaluation of medical records. The total number of peritonitis episodes was 56 cases, underwent by 30 adult patients on chronic peritoneal dialysis. Peritonitis was diagnosed according to ISPD recommendations. Causes and serious complications of peritoneal-related peritonitis were analyzed in every single year. Etiology of peritonitis was classified on the basis of the result of effluent dialysate culture as: gram-positive, gram-negative and culture negative. Peritoneal dialysis discontinuation or patient's death were defined as serious complications.

Results. Among 56 cases of peritoneal-related peritonitis $44.6 \%$ were gram-positive, $26.8 \%$ gram-negative and $28.6 \%$ culturenegative. No fungal or tuberculosis peritonitis were found. Because of the peritonitis complications in the evaluated period, six patients discontinued peritoneal dialysis and were switched to hemodialysis (20\%), two others died (6.7\%).

Conclusion. The further improvement in peritonitis' causes identification and treatment is needed in order to reduce number of serious complications in our medical center.
\end{abstract}

Keywords: peritoneal dialysis, peritonitis, etiology, serious complications.

DOI: $10.2478 /$ pjph-2018-0019

\section{INTRODUCTION}

Despite progress in dialysis treatment technique, peritoneal-related peritonitis is up to now a serious infectious complication of peritoneal dialysis (PD) [1]. Catheter loss or the patient's death are the most serious after-effects of peritonitis $[2,3]$. The quick and appropriate diagnosis of peritonitis symptoms is needed to avoid its complications. According to the definition given by the International Society of Peritoneal Dialysis (ISPD), to recognize peritonitis at least two of three following criteria are needed: clinical symptoms such as abdominal pain, fever; cloudy effluent with more than 100 white blood cells per $1 \mathrm{~mL}$ of dialysate, in which over $50 \%$ are neutrophils; identification of the pathogen in dialysate culture [4]. Peritonitis reasons differ geographically all around the world $[4,5]$. Most of the peritonitis are caused by bacteria, mainly gram-positive, especially by Staphylococci species: S. aureus and S. epidermidis [4-7]. Gram-negative peritonitis is less common, but its number is rising [4]. When compared gram-positive and gram-negative peritonitis, the latter one is responsible for more severe complications and is usually more difficult in the treatment, especially in Pseudomonas speciesrelated peritonitis [4-6,8]. Approximately twenty percent of dialysate culture worldwide are negative. These can be fungal, mycobacterial or even viral in their origin $[4,9]$. Some culturenegative peritonitis may be due to anaerobic pathogens or former antibiotics treatment $[10,11]$.

\section{AIM}

The present study was performed to make the analysis of the number, reasons and serious complications of peritonealrelated peritonitis, which occurred in the patients with endstage renal disease (ESRD) on peritoneal dialysis, treated in our medical center in the period 2012-2016.

\section{MATERIAL AND METHODS}

We performed a retrospective analysis of medical records of all PD patients in our medical center between 2012-2016 to find cases of peritonitis. To the current study there involved 30 adult patients on PD over 3 months, who underwent peritonitis. Peritonitis was defined according to the ISPD recommendations as presence of cloudy effluent, abdominal pain, white blood cells in effluent dialysate over $100>1 \mathrm{~mL}$ with $>50 \%$ neutrophils. We evaluated the number and causes of peritonitis 
in every single year of observation as well as serious complications of peritonitis. To confirmed peritonitis an effluent dialysate culture was performed. The results of dialysate-culture were taken from our database. The reasons of peritonitis were divided into: gram-positive, gram-negative and culture negative. Serious complications of peritonitis were defined as peritoneal dialysis discontinue or patients' death.

\section{RESULTS}

In retrospective 5-years study, we carefully analyzed all medical records of patients on PD in the years 2012-2016. The analysis revealed 56 cases of peritonitis in 30 individuals among all the patients on PD treatment. The characteristic of patients who underwent peritonitis is shown in Table 1. Total number of patients, who underwent peritonitis was 30 : 14 men aged from 20 to 76 years (mean age $51.93 \pm 13.83 \mathrm{y}$ ) and 16 women aged 24-75 years (mean age 51.56 $\pm 16.54 \mathrm{y}$ ). All patients suffered from ESRD. The reasons of ESRD were as follow: diabetic nephropathy $(10 \mathrm{pts} ; 5 \mathrm{M}, 5 \mathrm{~F})$, primary glomerulonephritis (7pts; 4M, 3F), interstitial nephropathy (7pts; $1 \mathrm{M}, 6 \mathrm{~F}$ ), vasculitis (3pts; $2 \mathrm{M}, 1 \mathrm{~F})$, autosomal dominant polycystic kidney disease $(1 \mathrm{M})$, other and unknown reasons $(2 \mathrm{M})$. All patients were treated for peritoneal dialysis for more than three months. Continued ambulatory peritoneal dialysis (CAPD) was used in 18 patients $(7 \mathrm{M}, 11 \mathrm{~F})$, automatic peritoneal dialysis (APD) was used in 9 patients $(6 \mathrm{M}, 3 \mathrm{~F})$ and continuous cyclic peritoneal dialysis (CCPD) was used in 3 patients (1M, 2F). Total number of peritonitis between 2012 and 2016 was 56 episodes. In detail, the number of the peritonitis episodes with their causes is shown in Table 2. The highest number of peritonitis episodes, that is 17 , was found in 2012. Then the episodes number systematically decreased, except the year 2016, in which 8 episodes were found. The lowest number of peritonitis was 6 and they occurred in 2015. Peritonitis jointly occurred in 30 individuals: 14 men and 16 women. Fourteen patients underwent only one episode of peritonitis $(6 \mathrm{M}, 8 \mathrm{~F})$, two episodes were observed in nine patients $(3 \mathrm{M}, 6 \mathrm{~F})$, three episodes were found in 4 patients $(3 \mathrm{M}, 1 \mathrm{~F})$, while four episodes were defined in 3 patients $(2 \mathrm{M}$, $1 \mathrm{~F})$.

According to the ISPD recommendation, in every case of peritonitis suspicion effluent dialysate culture was made. The analysis of cultures results revealed gram-positive bacteria as dominant cause of peritonitis in 2012 and 2014 (64.7\% and $66.7 \%$ respectively). Culture-negative peritonitis were found as dominant in the years 2013 and 2015 (50\%), while relatively high percentage of peritonitis caused by gram-negative bacteria was observed in the years 2015 and 2016 (33.3\% and $37.5 \%$ respectively). Details are shown in Table 2 .

Characteristic of the microorganisms responsible for peritonitis is shown in Table 3. In general, the most common peritonitis described in our center were gram-positive in their origin $(46.7 \%)$. The most common gram-positive bacteria were Staphylococcus species: S. aureus $(25 \%)$ and S. epidermidis (5.3\%). Surprisingly, among gram-negative bacteria peritonitis causes, Acinetobacter baumannii was the most common in our center (10.7\%). Among gram-negative bacteria Pseudomonas aeruginosa was found as second most frequent cause (5.3\%). Gram-negative peritonitis was observed jointly in $26.8 \%$, while culture-negative one in $28.6 \%$.
TABLE 1. The patients' characteristic.

\begin{tabular}{lcc}
\hline \hline & Men & Women \\
\hline Patients number & 14 & 16 \\
\hline Age (years) & $20-76$ & $24-75$ \\
\hline ESRD cause & & $(51.56 \pm 13.53)$ \\
\hline Diabetic nephropathy & 5 & 5 \\
\hline Primary GN & 4 & 3 \\
\hline Interstitial nephropathy & 1 & 6 \\
\hline Vasculitis & 1 & 2 \\
\hline ADPKD & 1 & - \\
\hline Another \& unknown & 2 & - \\
\hline Peritoneal dialysis type & & 11 \\
\hline CAPD & 7 & 3 \\
\hline APO & 6 & 2 \\
\hline CCPD & 1 & \\
\hline
\end{tabular}

Abbreviations: ESRD- end-stage renal disease, Primary GN- primary glomerulonephritis, ADPKD- autosomal dominant polycystic kidney disease, CAPD-continuous ambulatory peritoneal dialysis, APD- automatic peritoneal dialysis, CCPD- continuous cyclic peritoneal dialysiss.

TABLE 2. Total peritonitis number and their causes in particular years.

\begin{tabular}{cccccccc}
\hline \hline & $\begin{array}{c}\text { Total } \\
\text { peritonitis } \\
\text { number }\end{array}$ & $\begin{array}{c}\text { Gram (+) } \\
\text { peritonitis }\end{array}$ & $\%$ & $\begin{array}{c}\text { Gram (-) } \\
\text { peritonitis }\end{array}$ & $\begin{array}{c}\text { Culture- } \\
\text { negative } \\
\text { peritonitis }\end{array}$ & $\%$ \\
\hline 2012 & 17 & 11 & 64.7 & 4 & 23.5 & 2 & 11.8 \\
\hline 2013 & 16 & 4 & 25 & 4 & 25 & 8 & 50 \\
\hline 2014 & 9 & 6 & 66.7 & 2 & 22.2 & 1 & 11.1 \\
\hline 2015 & 6 & 1 & 16.7 & 2 & 33.3 & 3 & 50 \\
\hline 2016 & 8 & 3 & 37.5 & 3 & 37.5 & 2 & 25 \\
\hline Total & 56 & 25 & 44.6 & 15 & 26.8 & 16 & 28.6 \\
\hline
\end{tabular}

TABLE 3. The results of dialysate culture in particular years of the study.

\begin{tabular}{|c|c|c|c|c|c|c|c|}
\hline & 2012 & 2013 & 2014 & 2015 & 2016 & Total & $\%$ \\
\hline \multicolumn{8}{|c|}{ Gram-positive bacteria } \\
\hline S. aureus & 6 & 2 & 4 & - & 2 & 14 & 25 \\
\hline S. epidermidis & 2 & - & 1 & - & - & 3 & 5.4 \\
\hline S. haemolyticus & 1 & 1 & - & - & - & 2 & 3.5 \\
\hline Inne & - & 1 & 1 & 1 & 1 & 4 & 7.1 \\
\hline \multicolumn{8}{|c|}{ Gram-negative bacteria } \\
\hline $\begin{array}{l}\text { Acinetobacter } \\
\text { baumannii }\end{array}$ & 1 & 3 & - & 1 & 1 & 6 & 10.7 \\
\hline $\begin{array}{l}\text { Pseudomonas } \\
\text { aeruginosa }\end{array}$ & 1 & 1 & 1 & - & - & 3 & 5.4 \\
\hline Klebsiella spp. & 1 & - & - & - & 1 & 2 & 3.5 \\
\hline Enterobacteriaceae spp. & 3 & - & - & & - & 3 & 5.4 \\
\hline Morganella Morganii & - & - & - & 1 & 1 & 2 & 3.5 \\
\hline Serratia marcescens & - & & 1 & - & - & 1 & 1.8 \\
\hline $\begin{array}{l}\text { Culture-negative } \\
\text { peritonitis }\end{array}$ & 2 & 8 & 1 & 3 & 2 & 16 & 28.6 \\
\hline Total & 17 & 16 & 9 & 6 & 8 & 56 & \\
\hline
\end{tabular}


TABLE 4. The serious complications of peritonitis in particular years.

\begin{tabular}{lccccc}
\hline \hline & $\begin{array}{c}\text { Peritonitis } \\
\text { number }\end{array}$ & \multicolumn{2}{c}{ Catheter removal } & \multicolumn{2}{c}{ Patient's death } \\
\cline { 3 - 6 } & 17 & $\begin{array}{c}1 \\
(5.9 \%)\end{array}$ & Women & Men & Women \\
\hline 2012 & 16 & $\begin{array}{c}1 \\
(6.2 \%)\end{array}$ & & \\
\hline 2013 & 9 & $\begin{array}{c}2 \\
(22.2 \%)\end{array}$ & $\begin{array}{c}1 \\
(11.1 \%)\end{array}$ & $(11.1 \%)$ \\
\hline 2014 & 6 & & & 1 \\
\hline 2015 & 8 & & $(12.5 \%)$ & \\
\hline 2016 & & & & \\
\hline
\end{tabular}

The severe complications of peritonitis are shown in table 4 . As severe complication of peritonitis, we defined catheter removal and patients' death. Catheter removal was performed in 2 women and 4 men, while patient's death was observed in one man and one woman. In both women with catheter removal, diabetes mellitus was the reason of ESRD and in both $\mathrm{S}$. aureus was the peritonitis cause. One of these women underwent two episodes of peritonitis, while the second one, four episodes. In men, the need to remove peritoneal catheter occurred in 4 patients. Three of them were also diabetic and underwent 3 episodes (one patient), or 4 episodes of peritonitis (two patients). In two patients, S. aureus was the reason of peritonitis in the third one S. aureus followed by Acinetobacter baumannii. The fourth patient suffered from primary glomerulonephritis, underwent 2 episodes of peritonitis: first caused by S. aureus, second by Klebsiella pneumonie. Peritonitis was connected with patients' death in two cases: in one woman and one man. In woman, peritonitis was caused by unknown reason (culture-negative peritonitis) in man, the cause was Acinetobacter baumannii infection.

\section{DISCUSSION}

The highest number of peritonitis episodes was found in first two years of analysis (2012 and 2013) and was 17 and 16 episodes respectively, while in the last three years the number of peritonitis decreased to 6-9 episodes per year. The present analysis found gram-positive bacteria responsible for $44.6 \%$ of all peritonitis cases, gram-negative bacteria were responsible for $44.6 \%$ of peritonitis, and finally $28.6 \%$ peritonitis were found as culture-negative. Tian et al. found a similar distribution of peritonitis cases. They assessed gram-positive bacteria as the reason of the first peritonitis in $39.1 \%$ cases; only $17 \%$ of first peritonitis were gram-negative in the origin, while $40 \%$ of peritonitis were culture-negative [3]. In our study in 2012 and 2014 gram-positive bacteria were dominant as peritonitis reason $(64.7 \%$ and $66.7 \%$ respectively), while in the last two years of the study the percentage of gram-negative peritonitis was rising up to $33.3 \%$ in 2015 , and to $37.5 \%$ in 2016 . Similar results were published by Ren et al.: they analyzed 76 cases of peritonitis which occurred between 2008 and 2013, and found gram-positive peritonitis percentage decreased from $50 \%$ in 2008 to $36.2 \%$ in 2013 with parallel increase of gram-negative peritonitis: gram-negative peritonitis number increased from $34.4 \%$ in 2008 to $45.5 \%$ in 2013 [6]. As we found, among gram-positive peritonitis, $\mathrm{S}$ aureus was responsible for $25 \%$ of infections, this is in accordance with ISPS recommendation and some studies [4,7]. In a retrospective study, Higuchi et al. found S. aureus responsible for even more than $50 \%$ of peritonitis [1]. In total, S. aureus and S. epidermidis were responsible for $30.3 \%$ of all peritonitis in our center. As regards gram-negative peritonitis were $26.8 \%$ of all peritonitis cases in our center during the time of observation. This is higher value than currently published in ISPD data: gram-negative peritonitis should be approximately $15 \%$ of all [4]. But the trend is in accordance with presented by ISPS [4]. We observed the increase of gram-negative peritonitis during the time of the study, and found the highest percentage in 2015 (33.3\%) and in 2016 (37.5\%). The other studies also showed higher percentage of gram-negative peritonitis, e.g. $20.7 \%$ gram-negative peritonitis was noted by Higuchi et al. [1]. As most common gram-negative bacteria responsible for peritonitis, we found Acinetobacter baumannii in $10.7 \%$ cases and Pseudomonas aeruginosa $-5.3 \%$ of all peritonitis.

Culture-negative peritonitis were responsible in our center for $28.6 \%$ of all peritonitis cases. In 2012 and 2014 the percentage of culture-negative peritonitis was even lower than $20 \%$. This is in accordance with worldwide data [4]. We should be concerned about $50 \%$ of culture-negative peritonitis recognized in our center in 2013 and 2015. Almost as high percentage as in our study was found in Chinese one: in the years 2011-2013 culture-negative peritonitis were in $45 \%$ cases, while in 2008-2010 up to 34.4\% [6]. Similar results were described by Higuchi et al.: they found culture-negative peritonitis in $38.1 \%$ cases [1]. Also Tian et al. described culturenegative peritonitis as equal $40.1 \%$ [2]. All these results exceed ISPD recommendation [4]. It is worth to highlight, that among so-called "culture-negative" peritonitis, there are also anaerobic, viral, fungal, or tuberculosis in their origin $[1,4,5,10]$. Another reason of sterile dialysate culture (culture-negative) may be too short time of sample incubation, too small sample taken for incubation, or an appropriate way of taking the samples [4,9]. In some cases negative-culture peritonitis is caused by prior antibiotics treatment because of other reasons. Szeto et al. analyzed "sterile peritonitis" and found that in $45 \%$ of cases there were some technique problems with the samples, another $26 \%$ patients with sterile peritonitis were treated with antibiotics up to 30 days before peritonitis onset [11].

Less than a half of our patients (14 individuals - 46.7\%) underwent only one episode of peritonitis during the study period. The others underwent multiple peritonitis. We noticed, two episodes in 9 patients, three episodes in 4 patients and four episodes in 3 patients. Multiple peritonitis was described also in other studies [1]. As regards serious complications of peritonitis, six of our patients $(20 \%)$ were transferred to hemodialysis, while two patients $(6.7 \%)$ died because of peritonitis. These results are similar to described by Ren et al., who observed catheter loss in $15.8 \%$ patients with peritonitis and peritonitis-related death in $5.3 \%$ cases [4]. Study performed in 2013 in Japan revealed lower percentage of serious complication: the necessity of peritoneal dialysis discontinue in $13.6 \%$ cases and patient's death in only $1.1 \%$ [1]. In another study among six patients who underwent together 10 episodes of anaerobic peritonitis, one patient $(16.7 \%)$ lost the catheter, another one died (16.7\%) [10]. We also carefully analyzed reasons of catheter removal in each of six patients, who lost the peritoneal catheter. Two of patients were women: both diabetic and both underwent peritonitis caused by S. aureus. Two men, who underwent peritoneal catheter removal were diabetic, and peritonitis was connected with S. aureus infection. In total, four patients ended peritoneal dialysis because 
of S. aureus infection, which is considered to cause more severe forms of peritonitis [4]. Another man, who needed catheter removal, was also diabetic but the peritonitis was caused by Acinetobacter baumannii, gram-negative bacteria. The last man, who failure peritoneal dialysis technique, suffered from primary glomerulonephritis and Klebsiella pneumonia-related peritonitis. All patients described above were switched to hemodialysis. In two cases of peritonitis, patients died. One patient was a woman with ESRD because of interstitial nephritis with culture-negative peritonitis. The second patient, who died was a man suffering from vasculitis. In this man, peritonitis was caused by Acinetobacter baumannii. Obtained results are in opposite to those found in others' studies. In the published data, gram-negative, rather than gram-positive bacteria, are responsible for serious complications of peritonitis $[4,5,8]$.

\section{CONCLUSION}

Performed study revealed bacteria as main reason of peritonitis found in our PD center during a 5-years-observation - total $81.4 \%$. Gram-positive bacteria were responsible for $44.6 \%$ of all peritonitis cases. Another $26.8 \%$ peritonitis were gramnegative in their origin, while $26.8 \%$ were culture-negative. No fungal or tuberculous peritonitis were found. We observed a high number of serious peritonitis complications: in the evaluated period six patients discontinued peritoneal dialysis and were switched to hemodialysis $(20 \%)$, two others died $(6.7 \%)$. The further improvement in peritonitis' causes identification and treatment in our medical center is needed to reduce the number of serious peritonitis complications.

\section{REFERENCES}

1. Higuchi Ch, Ito M, Masakane I, Sakura H. Peritonitis in peritoneal dialysis patients in Japan: a 2013 retrospective questionnaire survey of Japanese Society for Peritoneal Dialysis member institutions. Renal Replacement Therapy. 2016;2:2.

2. Wang Z, Jiang L, Feng S, et al. Early peritonitis is an independent risk factor for mortality in elderly peritoneal dialysis patients. Kidney Blood Press Res. 2015;40:298-305.

3. Tian Y, Xie X, Xiang S, et al. Risk factors and outcomes of high peritonitis rate in continuous ambulatory peritoneal dialysis patients. Medicine. 2016; 5:49.

4. Burkard JM. Microbiology and therapy of peritonitis in continuous peritoneal dialysis. Official reprint from UpToDate www.uptodate.com (C)2017UpToDate ${ }^{\circledR}$

5. Akoh JA. Peritoneal dialysis associated infections: An update on diagnosis and management. World J Nephrol. 2012;1(4):106-22.

6. Ren W, Lan L, Jin Y, et al. Analysis of peritoneal dialysis-related peritonitis pathogenic bacteria and its drug-resistance. Int J Clin Exp Med. 2016;9(5):8648-55.

7. Travar M, Vlatkovic V, Vojvodic D. Microbiological aspects of peritonitis in patients on continuous ambulatory peritoneal dialysis: A monocentric five years follow up. J Infect Dis Ther. 2015;3:6.

8. Chao ChT, Lee SzY, Yang WS, et al. Citrobacter peritoneal dialysis peritonitis: Rare occurrence with poor diagnosis. Int J Med Sci. 2013;10.

9. de Freitas DG, Gokal R. Sterile peritonitis in the peritoneal dialysis patient. Perit Dial Int. 2005;25:146-51.

10. Chao ChT, Lee SzY, Yang WS, et al. Peritoneal dialysis peritonitis by anaerobic patogens: a retrospective case series. Nephrology. 2013;14:111

11. Szeto CC, Wong TY, Chow KM, et al. The clinical course of culturenegative peritonitis complicating peritoneal dialysis. Am J Kidney Dis. 2003;42:567-74

Corresponding author

Dr Agnieszka M. Grzebalska

Chair and Department of Nephrology, Medical University of Lublin

8 Jaczewskiego St., 20-954 Lublin, Poland

E-mail: AMGrzebalska@interia.pl

tel: +48817244537 